\title{
CLOPEN SETS IN HYPERSPACES ${ }^{1}$
}

\author{
PAUL BANKSTON
}

\begin{abstract}
Let $X$ be a space and let $H(X)$ denote its hyperspace (= all nonempty closed subsets of $X$ topologized via the Vietoris topology). Then $X$ is Boolean (= totally disconnected compact Hausdorff) iff $H(X)$ is Boolean; and if $B$ denotes the characteristic algebra of clopen sets in $X$ then the corresponding algebra for $H(X)$ is the free algebra generated by $B$ modulo the ideal which "remembers" the upper semilattice structure of $B$.
\end{abstract}

0 . Introduction. This note concerns the algebraic topology of the hyperspace $H(X)$ of a compact Hausdorff space $X$. Two antithetical situations immediately arise, namely when $X$ is connected (i.e., a continuum) and when $X$ is totally disconnected (i.e., a Boolean space). In the first situation one can study the homotopy of $H(X)$. Indeed, in 1931 Borsuk and Mazurkiewicz [2] showed that $H(X)$ is path connected if $X$ is metric; and in an unpublished paper, Banaschewski was able to remove the metrizability condition. Although the calculation of homotopy for $H(X)$ is still an open problem when $X$ is a general continuum (even a metric continuum), the question has long been settled for $X$ a Peano space (= locally connected metric continuum). In this case there is a beautiful succession of increasingly stronger results: $H(X)$ is Peano (Vietoris, 1923); $H(X)$ is contractible (Woydyslawski, 1938); $H(X)$ is an AR (Woydyslawski, 1939); and $H(X)$ is the Hilbert cube (Curtis and Schori, 1974). The historical details up to 1939 are in [2]; the last result is in [1]. Needless to say $H(X)$ has uninteresting homotopy when $X$ is Peano.

Our interest here lies in the second of the above situations, that is where $X$ is Boolean; and the algebraic object we study is the characteristic Boolean algebra $\chi(X)$ of clopen subsets of $X$. We then have a computational result which takes the following form: Let $B=\chi(X)$. Then $\chi(H(X))$ is the quotient of the free algebra generated by $B$ divided by the ideal which "remembers" the upper semilattice structure of $B$. Although the theorem makes sense without requiring that $H(X)$ be Boolean, the proof we present requires this property; and in fact it is easy to show (modulo classical results) that $X$ is Boolean iff $H(X)$ is Boolean as well.

\footnotetext{
Presented to the Society, November 15, 1974; received by the editors November 27, 1974.

AMS (MOS) subject classifications (1970). Primary 02J05, 54B20, 54F50, 54H10; Secondary 54F25, 55E05.

Key words and phrases. Hyperspace, Boolean space, Boolean algebra, clopen sets, characteristic algebra, Stone space.

${ }^{1}$ This work was carried out while the author was a Post-Doctoral Fellow (under the Canadian N.R.C.) at McMaster University, Hamilton, Ontario.
} 


\section{Preliminaries on hyperspaces.}

1.1 Definition. Let $X$ be a space, $S \subseteq X$. Define $S^{1}=\{C: C \subseteq X$ closed nonempty, $C \subseteq S\}$, and $S^{2}=\{C: C \subseteq X$ closed nonempty, $C \cap S \neq \varnothing\}$. The hyperspace $H(X)$ of $X$ consists of all closed nonempty subsets of $X$ topologized by taking subbasic sets of the form $U^{1}, U^{2}$ for $U \subseteq X$ open. The resulting topology is called the Vietoris topology.

An interesting and helpful fact is the following:

1.2 Lemma. Let $r=\left\{U_{1}, \ldots, U_{m}\right\}$ be a finite set of open subsets of $X$ and denote by $r \#$ the set $\left\{C \in H(X): C \subseteq U_{1} \cup \cdots \cup U_{m}\right.$, and for $1 \leqq k \leqq m$, $\left.C \cap U_{k} \neq \varnothing\right\}$. Then the collection of all such sets $r \#$ forms a basis for the Vietoris topology.

Proof. Let $r=\left\{U_{1}, \ldots, U_{m}\right\}, s=\left\{V_{1}, \ldots, V_{n}\right\}$, with $U=\cup r, V=\cup s$; and let $t$ be the set $\left\{U \cap V, U_{1} \cap V, \ldots, U_{m} \cap V, U \cap V_{1}, \ldots, U \cap V_{n}\right\}$. Then $t \#=r \# \cap s \#$, so the collection forms a basis for some topology $\tau$. Now if $U \subseteq X$ is open then $U^{1}=\{U\} \#, U^{2}=\{U, X\} \#$, whence $\tau$ contains the Vietoris topology. On the other hand if $r=\left\{U_{1}, \ldots, U_{m}\right\}$ then $r \#$ $=\left(U_{1} \cup \cdots \cup U_{m}\right)^{1} \cap U_{1}^{2} \cap \cdots \cap U_{m}^{2}$, so $\tau$ is in fact the Vietoris topology itself.

Remarks. (i) For metrizable $X$, say with metric $d, H(X)$ is metrizable as well via the well-known Hausdorff metric over $d$; and the Vietoris topology is precisely the derived metric topology.

(ii) Let $f: X \rightarrow Y$ be a continuous closed map and define $H(f): H(X)$ $\rightarrow H(Y)$ in the obvious way, i.e. $H(f)(C)=f^{\prime \prime} C=\{f(x): x \in C\}$. Then $H(f)$ is continuous, indeed

$$
H(f)^{-1}\left(\left\{U_{1}, \ldots, U_{m}\right\} \#\right)=\left\{f^{-1}\left(U_{1}\right), \ldots, f^{-1}\left(U_{m}\right)\right\} \# .
$$

$H(f)$ need not be a closed map, however.

Assume all spaces henceforth to be $T_{1}$. Then for each $X$ there is a natural injection $i: X \rightarrow H(X)$ taking $x \in X$ to its singleton $\{x\} . i$ is evidently a topological embedding (and in the metric case an isometry). Moreover, if $X$ is Hausdorff then $i$ is closed as well. For suppose $C \in H(X)-i^{\prime \prime} X$, with $a, b \in C$ distinct. Let $U=U(a), V=V(b)$ be a disjoint pair of open sets. Then $\{U, V, X\} \#$ is a nbd of $C$ missing $i^{\prime \prime} X$.

Now a standard result of Vietoris (see [4]) is that $X$ is compact Hausdorff iff $H(X)$ is compact Hausdorff. By extending this theorem we can easily prove that $X$ is Boolean iff $H(X)$ is Boolean. To see this, assume $H(X)$ is Boolean. Then, since $X$ embeds via $i$ as a closed subset of $H(X), X$ is Boolean as well. Conversely if $X$ is Boolean then $X$ has a basis of clopen sets so that if $C_{1}, C_{2} \in H(X)$ are distinct with, say, $a \in C_{1}-C_{2}$, then there is a clopen $U$ containing $a$ and missing $C_{2}$. Thus $\{U, X\} \#$ is a clopen nbd of $C_{1}$ missing $C_{2}$, proving that $H(X)$ is "ultra-Hausdorff" hence (in view of compactness) Boolean.

An alternative description of $H(X)$ for $X$ Boolean goes as follows (the straightforward details being left to the reader): Let $B$ be the algebra of clopen sets in $X$ and let $\sigma^{*}(B)$ be the set of proper filters in $B$. For $s=\left\{b_{1}, \ldots, b_{n}\right\}$ $\subseteq B$ let $s \#=\left\{p \in \sigma^{*}(B): b_{1} \vee \cdots \vee b_{n} \in p\right.$ and for $1 \leqq k \leqq n, b \in p$, 
$\left.b_{k} \wedge b \neq 0\right\}$. Then the sets $s \#$ form a topological basis; and the resulting space is precisely $H(X)$. The proof of this fact hinges upon the Stone duality between the closed nonempty subsets of a Boolean space and the proper filters of the corresponding Boolean algebra.

2. The main theorem. Let BTop denote the category of Boolean spaces and continuous maps with Boo the category of Boolean algebras and homomorphisms. Let

$$
\text { BTop } \underset{\sigma}{\stackrel{\chi}{\rightleftarrows}} \text { Boo }
$$

be the statement of Stone duality; where $\chi(X)$ is the characteristic algebra of clopen subsets of $X$, and $\sigma(B)$ is the Stone space of ultrafilters of $B$ topologized by taking as basis sets all sets of the form $b^{0}=\{u \in \sigma(B): b \in u\}$ as $b$ ranges over $B$. The Stone Duality Theorem says that $\chi$ and $\sigma$ are contravariant natural equivalences. Now if $X$ is compact, $Y$ is Hausdorff and $f: X \rightarrow Y$ continuous then $f$ is automatically a closed map. Thus in particular the hyperspace operator $H$ : BTop $\rightarrow$ BTop is functorial. A corollary of our theorem will be that there is an (algebraically defined) endofunctor $H^{\prime}$ : Boo $\rightarrow$ Boo which makes the category-theoretic diagram

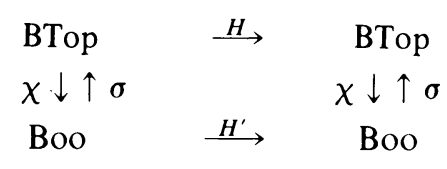

commutative.

We now define $H^{\prime}$. Let $B$ be a Boolean algebra. For ease of notation we will assume $B$ to be a field of sets and so use the usual set-theoretic notation for the Boolean algebraic operations. Now let $F(B)$ denote the free Boolean algebra generated by the elements of $B$. In this context we will use the connectives of elementary logic to denote the operations in $F(B)$ and use square brackets to distinguish the set $U$ in $B$ from its "name" $[U]$ in $F(B)$. So if $B=\chi(X)$ for some $X \in$ BTop then $\{\cup, \cap, X-(\cdot), \varnothing, X\}$ denote the Boolean operations in $B$, whereas $\{\vee, \wedge, \neg, 0,1\}$ denote the corresponding "formal" operations in $F(B)$. Typical elements of $F(B)$ include words of the form $[X],[\varnothing],[U],[U \cap(X-V)] \vee \neg[W]$, etc.

Given $B=\chi(X)$ we define the ideals $I_{1}, I_{2}$ in $F(B)$ as follows:

$I_{1}$ is generated by the words $\{[U] \wedge \neg[V]: U \subseteq V$ in $B\} \cup\{([U] \wedge[V])$ $\wedge \neg[U \cap V]: U, V \in B\} \cup\{[\varnothing], \neg[X]\} . I_{2}$ is generated by the words $\{[U] \wedge \neg[V]: U \subseteq V$ in $B\} \cup\{[U \cup V] \wedge \neg([U] \vee[V]): U, V \in B\} \cup$ $\{[\varnothing], \neg[X]\}$.

Intuitively $I_{1}$ (resp. $I_{2}$ ) "remembers" the lower (resp. upper) semilattice structure of $B$ so that $F(B) / I_{1}$, say, "believes" that $[U] \leqq[V]$ whenever $U$ $\subseteq V$, and that $[U] \wedge[V]=[U \cap V]$.

Now define the homomorphisms $h_{1}, h_{2}$ from $F(\chi(X))$ to $\chi(H(X))$ as follows: Let $U \in \chi(X)$. Then we set $f_{1}(U)=U^{1}, f_{2}(U)=U^{2}$. (Note. $U^{1}, U^{2}$ are clopen in $H(X)$ since $H(X)-U^{1}=(X-U)^{2}$, etc.) Since $F(\chi(X))$ is free, $f_{1}, f_{2}$ extend uniquely to homomorphisms $h_{1}, h_{2}$. We can now state our main theorem thusly: 
2.1 Theorem. Let $X \in$ BTop with $h_{1}, h_{2}: F(\chi(X)) \rightarrow \chi(H(X))$ given as above. Then:

(i) Both $h_{1}, h_{2}$ are epimorphisms.

(ii) $\operatorname{Ker} h_{1}=I_{1}$, Ker $h_{2}=I_{2}$ whence

$$
\chi(H(X)) \cong F(\chi(X)) / I_{1} \cong F(\chi(X)) / I_{2} .
$$

Thus if $B$ is any Boolean algebra, if we let $H^{\prime}(B)$ be either of the above quotients, and if $g: B_{1} \rightarrow B_{2}$ is a homomorphism, let $H^{\prime}(g)$ be the obvious quotient homomorphism. Then

(iii) The diagram

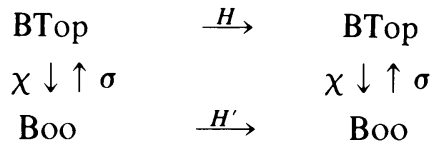

commutes (up to natural equivalences).

(iv) Let $j: F(\chi(X)) \rightarrow \chi(X)$ be the natural projection. Then $\operatorname{Ker} j=I_{1} \vee I_{2}$ $=$ the ideal generated by $I_{1} \cup I_{2}$.

Proof. (i) We show that the algebra $A$ generated by the sets $U^{1}, U$ $\in \chi(X)$, is all of $\chi(H(X))$. Indeed by compactness the sets $s \#$ form a basis for $H(X)$ as $s$ ranges over the finite subsets of $\chi(X)$. Also since

$$
s \#=\left\{U_{1}, \ldots, U_{m}\right\} \#=\left(U_{1} \cup \cdots \cup U_{m}\right)^{1} \cap\left(U_{1}^{2} \cap \cdots \cap U_{m}^{2}\right)
$$

we have that the $U^{1}$ 's generate a basis for the Vietoris topology. Since every clopen set in $H(X)$ is compact and is a union of elements from $A$, it is a union of finitely many elements from $A$ and is thus itself in $A$. Thus $A=\chi(H(X))$.

(ii) Since $\varnothing^{1}=\varnothing, X^{1}=X, U^{1} \subseteq V^{1}$ for $U \subseteq V$, and $(U \cap V)^{1}=U^{1}$ $\cap V^{1}$ for all $U, V \in \chi(X)$, we have $\operatorname{Ker} h_{1} \supseteq I_{1}$. Similarly $\operatorname{Ker} h_{2} \supseteq I_{2}$. Let $w \in F(\chi(X))$ and assume $w$ is represented as a disjunction of conjunctions of generators and complements of generators. Such a conjunction we refer to as a minterm. If $w=w_{1} \vee \cdots \vee w_{n}$ is a disjunction of minterms, and if the minterms of $\operatorname{Ker} h_{1}$ are in $I_{1}$ then $\operatorname{Ker} h_{1} \subseteq I_{1}$. For $w \in \operatorname{Ker} h_{1} \Rightarrow w_{k}$ $\in \operatorname{Ker} h_{1}$, each $1 \leqq k \leqq n$. Thus $w \in I_{1}$. So it suffices to prove the inclusion for minterms, of which there are three kinds: positive (only unnegated generators occur), negative, and mixed.

Positive. $\quad w=\left[U_{1}\right] \wedge \cdots \wedge\left[U_{m}\right] \in \operatorname{Ker} h_{1}$. Then $U^{1} \cap \cdots \cap U_{m}^{1}=$ $\left(U_{1} \cap \cdots \cap U_{m}\right)=\varnothing$ iff $U_{1} \cap \cdots \cap U_{m}=\varnothing$. Now

$$
\begin{aligned}
\left(\left[U_{1}\right]\right. & \left.\wedge \cdots \wedge\left[U_{m}\right]\right) \wedge \neg\left[U_{1} \cap \cdots \cap U_{m}\right] \\
& =\left[U_{1}\right] \wedge \cdots \wedge\left[U_{m}\right] \wedge \neg[\varnothing] \in I_{1} .
\end{aligned}
$$

But $[\varnothing] \in I_{1}$, so $\left[U_{1}\right] \wedge \cdots \wedge\left[U_{m}\right] \in I_{1}$ as well.

Negative. $w=\neg\left[V_{1}\right] \wedge \cdots \wedge \neg\left[V_{n}\right] \in \operatorname{Ker} h_{1}$. Then $\left(X-V_{1}\right)^{2} \cap \cdots$ $\cap\left(X-V_{n}\right)^{2}=\varnothing$ iff some $V_{k}=X$. But $\neg[X] \in I_{1}$, so $w \in I_{1}$ too.

Mixed. $w=\left[U_{1}\right] \wedge \cdots \wedge\left[U_{m}\right] \wedge \neg\left[V_{1}\right] \wedge \cdots \wedge \neg\left[V_{n}\right] \in \operatorname{Ker} h_{1}$. Then

$$
\left(U_{1} \cap \cdots \cap U_{m}\right)^{1} \cap\left(X-V_{1}\right)^{2} \cap \cdots \cap\left(X-V_{n}\right)^{2}=\varnothing
$$


whence $U_{1} \cap \cdots \cap U_{m} \subseteq V_{l}$ for some $1 \leqq l \leqq n$. Thus $\left[U_{1} \cap \cdots \cap U_{m}\right]$ $\wedge \neg\left[V_{l}\right] \in I_{1}$ so $\left(\left[U_{1}\right] \wedge \cdots \wedge\left[U_{m}\right]\right) \wedge \neg\left[V_{l}\right] \in I_{1}$ and therefore $w \in I_{1}$. The proof that Ker $h_{2}=I_{2}$ is similar.

(iii) This follows straightforwardly from (ii).

(iv) This is proved in the same way as (ii).

\section{REFERENCES}

1. D. Curtis and R. Schori, $2^{x}$ and $C(X)$ are homeomorphic to the Hilbert cube, Bull. Amer. Math. Soc. 80 (1974), 927-931.

2. J. L. Kelley, Hyperspaces of a continuum, Trans. Amer. Math. Soc. 52 (1942), 22-36. MR 3, 315.

3. J. Nagata, Modern dimension theory, Bibliotheca Math., vol. 6, Interscience, New York, 1965. MR $34 \# 8380$.

4. K. Kuratowski, Topology. Vol. 1, 5th ed., PWN, Warsaw; Academic Press, New York, 1966. MR $36 \# 840$.

Department of Mathematics, McMaster University, Hamilton, Ontario, Canada 